Pacific Journal of Mathematic 


\title{
A - P CONGRUENCES ON BAER SEMIGROUPS
}

\author{
B. J. THORNE
}

In this paper a coordinatizing Baer semigroup is used to pick out an interesting sublattice of the lattice of congruence relations on a lattice with 0 and 1 . These congruences are defined for any lattice with 0 and 1 and have many of the nice properties enjoyed by congruence relations on a relatively complemented lattice.

These results generalize the work of S. Maeda on Rickart (Baer) rings and are related to G. Gratzer and E. T. Schmidt's work on standard ideals.

In [7] M. F. Janowitz shows that lattice theory can be approached by means of Baer semigroups. A Baer semigroup is a multiplicative semigroup $S$ with 0 and 1 in which the left and right annihilators, $L(x)=\{y \in S: y x=0\}$ and $R(x)=\{y \in S: x y=0\}$, of any $x \in S$ are principal left and right ideals generated by idempotents. For any Baer semigroup $S, \mathscr{L}(S)=\{L(x): x \in S\}$ and $\mathscr{R}(S)=\{R(x): x \in S\}$, ordered by set inclusion, are dual isomorphic lattices with 0 and 1 . The Baer semigroup $S$ is said to coordinatize the lattice $L$ if $\mathscr{L}(S)$ is isomorphic to $L$. The basic point is Theorem 2.3, p. 1214 of [7], which states: a partially ordered set $P$ with 0 and 1 is a lattice if and only if it can be coordinatized by a Baer semigroup.

It will be convenient to introduce the convention that $S$ will always denote a Baer semigroup and that for any $x \in S, x^{l}$ and $x^{r}$ will denote idempotent generators of $L(x)$ and $R(x)$ respectively. Also the letters $e, f, g$, and $h$ shall always denote idempotents of $S$.

Some background material is presented in $\S 1$. In $\S 2, A-P$ congruences are defined and it is shown that every $A-P$ congruence $\rho$ on $S$ induces a lattice congruence $\Theta_{\rho}$ on $\mathscr{L}(S)$ such that $\mathscr{L}(S) / \Theta_{\rho} \cong$ $\mathscr{L}(S / \rho)$. In $\S 3$ congruences which arise in this manner are characterised as the set of all equivalence relations on $\mathscr{C}(S)$ which are compatible with a certain set of maps on $\mathscr{L}(S)$. These congruences are called compatible with $S$. They are standard congruences and are thus determined by their kernels.

The ideals of $\mathscr{L}(S)$ which are kernels of congruences compatible with $S$ are characterised in $\S 4$. In $\S 5$ it is shown that a principal ideal, [(0), $S e]$, is the kernel of a congruence compatible with $S$ if and only if $e$ is central in $S$. In $\S 6$ this is applied to complete Baer semigroups to show that, in this case, the congruence compatible with $S$ form a Stone lattice. 
1. Preliminaries. We shall let $L(M)=\{y \in S: y x=0$ for all $x \in M\}$ and $R(M)=\{y \in S: x y=0$ for all $x \in M\}$ for any set $M \leqq S$. The following is a summary of results found on pp. 85-86 of [8].

LEMMA 1.1. Let $x, y \in S$.

( i ) $x S \subseteq y S$ implies $L(y) \subseteq L(x)$; $S x \leqq S y$ implies $R(y) \leqq R(x)$.

( ii ) $S x \leqq L R(x) ; x S \subseteq R L(x)$.

(iii) $L(x)=L R L(x) ; R(x)=R L R(x)$.

(iv) $S x \in \mathscr{L}(S)$ if and only if $S x=L R(x) ; x S \in \mathscr{R}(S)$ if and only if $x S=R L(x)$.

( v) The mappings $e S \rightarrow L(e S)$ and $S f \rightarrow R(S f)$ are mutually inverse dual isomorphisms between $\mathscr{R}(S)$ and $\mathscr{L}(S)$.

(vi) Let $S e, S f \in \mathscr{L}(S)$ and $S h=L\left(e f^{r}\right)$. Then he $=(h e)^{2}$, Se $\cap S f=S h e \in \mathscr{L}(S)$, and $S e \bigvee S f=L\left(e^{r} S \cap f^{r} S\right)$.

(vii) Let $e S, f S \in \mathscr{R}(S)$ and $g S=R\left(f^{l} e\right)$. Then $e g=(e g)^{2}$, $e S \cap f S=e g S \in \mathscr{R}(S)$, and $e S \vee f S=R\left(S e^{l} \cap S f^{l}\right)$.

Note that the meet operation in $\mathscr{L}(S)$ and $\mathscr{R}(S)$ is set intersection and that the trivial ideals, $S$ and $(0)$, are the largest and smallest elements of both $\mathscr{L}(S)$ and $\mathscr{R}(S)$.

We shall be interested in a class of isotone maps introduced by Croisot in [2].

Definition 1.2. Let $P$ be a partially ordered set. An isotone map $\phi$ of $P$ into itself is called residuated if there exists an isotone map $\phi^{+}$of $P$ into $P$ such that for any $p \in P, p \phi^{+} \phi \leqq p \leqq p \phi \phi^{+}$. In this case $\phi^{+}$is called a residual map.

Clearly $\phi^{+}$is uniquely determined by $\phi$ and conversly. The pair $\left(\dot{\phi}, \phi^{+}\right)$sets up a Galois connection between $P$ and its dual. Thus we can combine results from [2], [3], and [11] to get.

LEMMA 1.3. Let $P$ be a partially ordered set and $\phi$ and $\psi$ maps of $P$ into itself.

(i) If $\phi$ and $\psi$ are residuated then $\phi \psi$ is residuated and $(\phi \psi)^{+}=\psi^{+} \phi^{+}$.

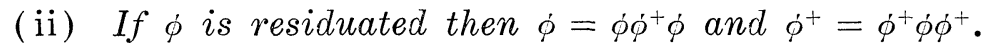

(iii) Let $\phi$ be residuated and $\left\{x_{\alpha}\right\}$ be any family of elements of P. If $\mathbf{V}_{\alpha} x_{\alpha}$ exists then $\mathbf{V}_{\alpha}\left(x_{\alpha} \phi\right)$ exists and $\mathbf{V}_{\alpha}\left(x_{\alpha} \phi\right)=\left(\mathbf{V}_{\alpha} x_{\alpha}\right) \dot{\phi}$. Dually if $\Lambda_{\alpha} x_{\alpha}$ exists then $\Lambda_{\alpha}\left(x_{\alpha} \phi^{+}\right)$exists and $\Lambda_{\alpha}\left(x_{\alpha} \phi^{+}\right)=\left(\Lambda_{\alpha} x_{\alpha}\right) \phi^{+}$.

(iv) $A$ necessary and sufficient condition that $\phi$ be residuated is that for any $x \in L,\{z: z \phi \leqq x\}$ has a largest element $x^{*}$. In this case $\phi^{+}$is given by $x \dot{\phi}^{+}=x^{*}$. 
According to Lemma 1.3 (i) the set of residuated maps forms a semigroup for any partially ordered set $P$. We shall denote the semigroup of residuated maps on $P$ by $S(P)$. In [7], Theorem 2.3, p. 1214, it is shown that $P$ is a lattice if and only if $S(P)$ is a Baer semigroup. In this case $S(P)$ coordinatizes $P$.

In [8], pp. 93, 94, it is shown that any Baer semigroup $S$ can be represented as a semigroup of residuated maps on $\mathscr{L}(S)$. We shall be interested in the maps introduced to achieve this.

Lemma 1.4. For any $x \in S$ define $\phi_{x}: \mathscr{L}(S) \rightarrow \mathscr{L}(S)$ by $S e \phi_{x}=$ $L R(e x)$.

(i ) $\phi_{x}$ is residuated with residual $\phi_{x}^{+}$given by $S e \phi_{x}^{+}=L\left(x e^{r}\right)$.

(ii) If $L R(y)=$ Se then $S e \dot{\phi}_{x}=L R(y x)$.

(iii) Let $S_{0}=\left\{\phi_{x}: x \in S\right\}$. Then $S_{0}$ is a Baer semigroup which coordinatizes $\mathscr{L}(S)$.

(iv) The $\operatorname{map} x \rightarrow \phi_{x}$ is a homomorphism, with kernel $\{0\}$, of $S$ into $S_{0}$.

We shall now develop an unpublished result due to D. J. Foulis and M. F. Janowitz.

Definition 1.5. A semigroup $S$ is a complete Baer semigroup if for any subset $M$ of $S$ there exist idempotents $e, f$ such that $L(M)=S e$ and $R(M)=f S$.

In proving Lemma 2.3 of [7] the crucial observation was [7] Lemma 2.1, p. 1213, where it is shown that for any lattice $L$ and any $a \in L$ there are idempotent residuated maps $\theta_{a}$ and $\psi_{a}$ given by:

$$
x \theta_{a}=\left\{\begin{array}{cc}
x & x \leqq a \\
a & \text { otherwise }
\end{array} \quad x \psi_{a}=\left\{\begin{array}{cc}
0 & x \leqq a \\
x \vee a & \text { otherwise }
\end{array}\right.\right.
$$

Theorem 1.6. Let $P$ be a partially ordered set with 0 and 1. Then the following conditions are equivalent.

(i) $P$ is a complete lattice.

(ii) $S(P)$ is a complete Baer semigroup.

(iii) $P$ can be coordinatized by a complete Baer semigroup.

Proof. (i) $\Rightarrow$ (ii) Let $P$ be a complete lattice and $M \subseteq S(P)$ with $m=\mathbf{V}\{1 \phi: \phi \in M\}$ and $n=\Lambda\left\{0 \phi^{+}: \phi \in M\right\}$. It is easily verified that $L(M)=S(P) \theta_{n}$ and $R(M)=\psi_{m} S(P)$.

(ii) $\Rightarrow$ (iii) follows from [7], Theorem 2.3.

(iii) $\Rightarrow$ (i) Let $S$ be a complete Baer semigroup coordinatizing $P$ and $\mathscr{P}(S)$ the complete lattice of all subsets of $S$. Define $\alpha$ and $\beta$ 
mapping $\mathscr{P}(S)$ into $\mathscr{P}(S)$ by $M \alpha=L(M)$ and $M \beta=R(M)$. Clearly $(\alpha, \beta)$ sets up a Galois connection of $\mathscr{P}(S)$ with itself. Since $S$ is a complete Baer semigroup $\mathscr{L}(S)$ is the set of Galois closed objects of $(\alpha, \beta)$. Thus $\mathscr{L}(S)$ is a complete lattice.

We conclude this section with some relatively well known facts about lattice congruences. An equivalence relation $\Theta$ on a lattice is a lattice congruence if $a \Theta b$ and $c \Theta d$ imply $(a \bigvee c) \Theta(b \bigvee d)$ and $(a \wedge c) \Theta(b \wedge d)$. We shall sometimes write $a \equiv b(\Theta)$ in place of $a \Theta b$. With respect to the order $\Theta \leqq \Theta^{\prime}$ if and only if $a \Theta b$ implies $a \Theta^{\prime} b$, the set of all lattice congruences on a lattice $L$ is a complete lattice, denoted by $\Theta(L)$, with meet and join given as follows :

THEOREM 1.7. Let $L$ be a lattice and $\Gamma$ a subset of $\Theta(L)$.

(i) $a \equiv b(\Lambda \Gamma)$ if and only if $a \gamma b$ for all $\gamma \in \Gamma$.

(ii) $a \equiv b(\bigvee \Gamma)$ if and only if there exist finite sequences $a_{0}, a_{1}, \cdots, a_{n}$ of elements of $L$ and $\gamma_{1}, \cdots, \gamma_{n}$ of elements of $\Gamma$, such that $a=a_{0}, a_{n}=b$, and $a_{i-1} \gamma_{i} a_{i}$ for $i=1, \cdots, n$.

The largest element $\iota$ of $\Theta(L)$ is given by $a \iota b$ for all $a, b \in L$ and the smallest element $\omega$ is given by $a \omega b$ if and only if $a=b$.

In [4] it is shown that $\Theta(L)$ is distributive. In fact we have :

THEOREM 1.8. Let $L$ be a lattice. The $\Theta(L)$ is a distributive lattice such that for any family $\left\{\Theta_{\alpha}\right\} \leqq \Theta(L)$

$$
\left(\mathbf{V}_{\alpha} \Theta_{\alpha}\right) \Lambda \Psi=\mathbf{V}_{\alpha}\left(\Theta_{\alpha} \Lambda \Psi\right)
$$

for any $\Psi \in \Theta(L)$.

Thus by Theorem 15, p. 147, of [1] we have:

Theorem 1.9. For any lattice $L, \Theta(L)$ is pseudo-complemented.

Finally we mention that if $\Theta \in \Theta(L)$ then $a \Theta b$ if and only if $x \Theta y$ for all $x, y \in[a \Lambda b, a \bigvee b]$.

2. A-P congruences. In [10] $\mathrm{S}$. Maeda defines annihilator preserving homomorphisms for rings. We shall take the same definition for semigroups with 0 .

Definition 2.1. A homomorphism $\phi$ of a semigroup $S$ with 0 is called an annihilator preserving $(A-P)$ homomorphism if for any $x \in S, R(x) \dot{\phi}=R(x \dot{\phi}) \cap S \dot{\phi}$ and $L(x) \dot{\phi}=L(x \dot{\phi}) \cap S \dot{\phi}$. A congruence relation $\rho$ on a semigroup $S$ is called an $A-P$ congruence if the natural 
homomorphism induced by $\rho$ is an $A-P$ homomorphism.

For any congruence $\rho$ on a semigroup $S$ and any $x \in S$ let $x / \rho$ denote the equivalence class of $S / \rho$ containing $x$. Similarly for any set $A \cong S$, let $A / \rho=\{x / \rho \in S / \rho: x \in A\}$. If $S$ has a 0 then $R(x) / \rho \subseteq$ $R(x / \rho)$ and $L(x) / \rho \subseteq L(x / \rho)$. Thus a congruence $\rho$ is an $A-P$ congruence if and only if $R(x / \rho) \subseteq R(x) / \rho$ and $L(x / \rho) \subseteq L(x) / \rho$. Note that we are using $L$ and $R$ to denote the left and right annihilators both in $S$ and in $S / \rho$.

Theorem 2.2. Let $\rho$ be an $A-P$ congruence on a semigroup $S$. If $e$ and $f$ are idempotents of $S$ such that $S e=L(x)$ and $f S=R(y)$ for some $x, y \in S$, then $(S / \rho)(e / \rho)=L(x / \rho)$ and $(f / \rho)(S / \rho)=R(y / \rho)$. Thus if $S$ is a Baer semigroup so is $S / \rho$.

Proof. Since $\rho$ is an $A-P$ congruence $L(x / \rho)=L(x) / \rho$. Thus $L(x)=S e$ gives $L(x / \rho)=L(x) / \rho=(S e) / \rho=(S / \rho)(e / \rho)$. Similarly $R(x)=$ $f S$ gives $R(x / \rho)=(f / \rho)(S / \rho)$.

We now use an $A-P$ congruence $\rho$ on $S$ to induce a homomorphism of $\mathscr{L}(S)$ onto $\mathscr{L}(S / \rho)$.

Theorem 2.3. Let $\rho$ be an $A-P$ congruence on $S$. Then $\theta_{\rho}: \mathscr{L}(S) \rightarrow \mathscr{L}(S / \rho)$ by $L(x) \theta_{\rho}=L(x / \rho)$ is a lattice homomorphism of $\mathscr{L}(S)$ onto $\mathscr{L}(S / \rho)$.

Proof. Let $S e, S f \in \mathscr{L}(S)$ and note that, by Theorem 2.2,

$$
S e \theta_{\rho}=(S / \rho)(e / \rho) \text { and } S f \theta_{\rho}=(S / \rho)(f / \rho) \text {. }
$$

Clearly $\theta_{\rho}$ is well defined since if $L(x)=L(y)$ then

$$
L(x / \rho)=L(x) / \rho=L(y) / \rho=L(y / \rho) .
$$

By Lemma 1.1 (vi), She $=S e \cap S f$ where $S h=L\left(e f^{r}\right)$. Applying Theorem 2.2 gives $\left(f^{r} / \rho\right)(S / \rho)=R(f / \rho)$ and $(S / \rho)(h / \rho)=L\left((e / \rho)\left(f^{r} / \rho\right)\right)$. Thus applying Lemma 1.1 (vi) to $S / \rho$ yields

$$
(S / \rho)(e / \rho) \cap(S / \rho)(f / \rho)=(S / \rho)(h / \rho)(e / \rho)=(S / \rho)(h e / \rho) .
$$

Therefore, $S e \theta_{\rho} \cap S f \theta_{\rho}=(S e \cap S f) \theta_{\rho}$. By a dual argument $\theta_{\rho}^{*} \mathscr{R}(S) \rightarrow$ $\mathscr{R}(S / \rho)$ by $R(x) \theta_{\rho}^{*}=R(x / \rho)$ is also a meet homomorphism.

By Lemma 1.1 (vi) $S e \bigvee S f=L(R(e) \cap R(f))$. Let $g S=R(e) \cap R(f)$ so that $S e \bigvee S f=S g^{l}$. Since $\theta_{\rho}^{*}$ is a meet homomorphism,

$$
R(e / \rho) \cap R(f / \rho)=(g / \rho)(S / \rho) \text {. }
$$


Noting that $L(g / \rho)=(S / \rho)\left(g^{l} / \rho\right)$ and applying Lemma 1.1 (vi) to $S / \rho$ gives

$$
(S / \rho)(e / \rho) \bigvee(S / \rho)(f / \rho)=(S / \rho)\left(g^{l} / \rho\right)
$$

Thus $S e \theta_{\rho} \bigvee S f \theta_{\rho}=(S e \bigvee S f) \theta_{\rho}$ and $\theta_{\rho}$ is a lattice homomorphism. Clearly $\theta_{\rho}$ is onto.

For any $A-P$ congruence $\rho$ on $S$ let $\Theta_{\rho}$ denote the lattice congruence $\theta_{\rho} \circ \theta_{\rho}^{-1}$ induced on $\mathscr{L}(S)$ by $\theta_{\rho}$.

CoRollary 2.4. $\mathscr{L}(S) / \Theta_{\rho} \cong \mathscr{L}(S / \rho)$.

3. Compatible congruences. In this section we shall characterise lattice congruence which are induced by an $A-P$ congruence on a coordinatizing Baer semigroup in the manner given in Theorem 2.3. Since $L \cong \mathscr{L}(S)$ for any Baer semigroup $S$ coordinatizing $L$, we shall lose no generality by considering only lattices of the form $\mathscr{L}(S)$.

The residuated maps $\phi_{x}, x \in S$, defined in Lemma 1.4, play a central role in the theory of Bear semigroups. We shall be interested in equivalence relations on $\mathscr{L}(S)$ which are compatible with $\phi_{x}$ and $\phi_{x}^{+}$, considered as unary operations on $\mathscr{L}(S)$.

Definition 3.1. An equivalence relation $E$ on $\mathscr{L}(S)$ is called compatible with $S$ if for any $x \in S$,

$$
S e E S f \Rightarrow\left(S e \phi_{x}\right) E\left(S f \dot{\phi}_{x}\right) \quad \text { and } \quad\left(S e \phi_{x}^{+}\right) E\left(S f \phi_{x}^{+}\right) .
$$

By [7] Lemma 3.1 and 3.2, pp. 1214-1215, Se $\cap S f=S e \cap S \phi_{f}=S e \phi_{f}^{+} \phi_{f}$. Dually $S e \mathrm{~V} S f=S e \phi_{f r} \dot{\phi}_{f}^{+}$. Thus we have:

LEMMA 3.3. Any equivalence relation compatible with $S$ is a lattice congruence.

We now consider an $A-P$ congruence $\rho$ on $S$ and $\Theta_{\rho}$, the lattice congruence induced on $\mathscr{L}(S)$ by $\rho$ as in Theorem 2.3.

Theorem 3.4. Let $\rho$ be an $A-P$ congruence on $S$. Then $\Theta_{\rho}$ is compatible with $S$.

Proof. Since $S e \Theta_{\rho} S f$ if and only if $(S / \rho)(e / \rho)=(S / \rho)(f / \rho), S e \Theta_{\rho} S f$ implies $(e / \rho)=(e / \rho)(f / \rho)$ and $(f / \rho)=(f / \rho)(e / \rho)$. Note that for any $y \in S, L R(y) \theta_{\rho}=L R(y / \rho)$. If $S e \Theta_{\rho} S f$ we have 


$$
\begin{aligned}
\left(S e \phi_{x}\right) \theta_{\rho} & =L R((e x) / \rho)=L R((e / \rho)(x / \rho)) \\
& =L R((e / \rho)(f / \rho)(x / \rho)) \leqq L R((f / \rho)(x / \rho))=\left(S f \phi_{x}\right) \theta_{\rho} .
\end{aligned}
$$

By symmetry $\left(S f \phi_{x}\right) \theta_{\rho}=\left(S e \phi_{x}\right) \theta_{\rho}$ ie. $S e \phi_{x} \Theta_{\rho} S f \phi_{x}$.

Now $R(e / \rho)=R((e / \rho)(f / \rho)) \supseteqq R(f / \rho)=R((f / \rho)(e / \rho)) \supseteqq R(e / \rho)$. Thus $\left(e^{r} / \rho\right)(S / \rho)=\left(f^{r} / \rho\right)(S / \rho)$. But

$$
\left(S e \phi_{x}^{+}\right) \theta_{\rho}=L\left(x e^{r}\right) \theta_{\rho}=L\left(\left(x e^{r}\right) / \rho\right)=L\left((x / \rho)\left(e^{r} / \rho\right)\right)
$$

and similarly $\left(S f \phi_{x}^{+}\right) \theta_{\rho}=L\left((x / \rho)\left(f^{r} / \rho\right)\right)$. Clearly $y / \rho \in L\left((x / \rho)\left(e^{r} / \rho\right)\right)$ if and only if $(y / \rho)(x / \rho) \in L\left(\left(e^{r} / \rho\right)(S / \rho)\right)=L\left(\left(f^{r} / \rho\right)(S / \rho)\right)$. Thus we have $\left(S e \phi_{x}^{+}\right) \theta_{\rho}=\left(S f \phi_{x}^{+}\right) \theta_{\rho}$. Therefore, $S e \phi_{x}^{+} \Theta_{\rho} S f \phi_{x}^{+}$and $\Theta_{\rho}$ is compatible with $S$.

By the following theorem every congruence compatible with $S$ is determined by its kernel in a very nice way.

THEOREm 3.5. Let $\Theta$ be a congruence compatible with $S$. Then the following are equivalent.

(i) $S e \Theta S f$.

(ii) $S e \phi_{f} \vee \mathrm{Sf} \phi_{e^{r}} \in \operatorname{ker} \Theta$.

(iii) There is an $S g \in \operatorname{ker} \Theta$ such that $S e \bigvee S f=S e \bigvee S g=$ $S f \vee S g$.

Proof (i) $\Rightarrow$ (ii) Since $\Theta$ is compatible with $S$, $S e$ $9 f$ gives $S e \dot{\phi}_{f} \vartheta S f \dot{\phi}_{f} r=(0)$, i.e., $S e \dot{\phi}_{f} \in \operatorname{ker} \Theta$. By symmetry $S f \dot{\phi}_{e^{r}} \in \operatorname{ker} \Theta$ so we have (ii).

(ii) $\Rightarrow$ (iii) Let $S g=S e \phi_{f^{r}} \bigvee S f \phi_{e^{r}} \in \operatorname{ker} \Theta$ and claim $S e \bigvee S g=$ $S f \vee S g$, i.e.,

$$
L R(e) \bigvee L R\left(e f^{r}\right) \bigvee L R\left(f e^{r}\right)=L R(f) \bigvee L R\left(e f^{r}\right) \bigvee L R\left(f e^{r}\right)
$$

By Lemma 1.1 (v) this is equivalent to

$$
R(e) \cap R\left(e f^{r}\right) \cap R\left(f e^{r}\right)=R(f) \cap R\left(e f^{r}\right) \cap R\left(f e^{r}\right) .
$$

Let $x \in R(e) \cap R\left(e f^{r}\right) \cap R\left(f e^{r}\right)$. Then $x=e^{r} x$ and $f x=f e^{r} x=0$ so $x \in R(f) \cap R\left(e f^{r}\right) \cap R\left(f e^{r}\right)$. By symmetry

$$
R(e) \cap R\left(e f^{r}\right) \cap R\left(f e^{r}\right)=R(f) \cap R\left(e f^{r}\right) \cap R\left(f e^{r}\right)
$$

so we have $S e \mathrm{~V} S g=S f \bigvee S g$. To show that $S e \bigvee S f=S e \bigvee S g=$ $S f \bigvee S g$ we need only show that $S g \subseteq S e \bigvee S f$. This is equivalent to $R(e) \cap R(f) \subseteq R\left(e f^{r}\right) \cap R\left(f e^{r}\right)$. But if $x \in R(e) \cap R(f)$ then $x=e^{r} x=$ $f^{r} x$, so $e f^{r} x=e x=0$ and $f e^{r} x=f x=0$, i.e., $x \in R\left(e f^{r}\right) \cap R\left(f e^{r}\right)$.

(iii) $\Rightarrow$ (i) If $S e \bigvee S g=S f \bigvee S g$ and $S g \vartheta(0)$ then $S \otimes \Theta S e \bigvee S g=$ $S f \bigvee S g \otimes S f$. 
A congruence $\Theta$ on a lattice is called standard if there is an ideal $S$ such that $a \Theta b$ if and only if $a \bigvee b=(a \Lambda b) \bigvee s$ for some $s \in S$.

CoROllary 3.6. Any congruence $\Theta$ compatible with $S$ is a standard congruence.

Proof. Since $\Theta$ is a lattice congruence $S e \Theta S f$ if and only if $(S e \bigvee S f) \Theta(S e \cap S f)$. By Theorem 3.5 this is equivalent to

$$
(S e \bigvee S f) \bigvee(S e \cap S f)=S e \bigvee S f=(S e \cap S f) \bigvee S g
$$

for some $S g \in \operatorname{ker} \Theta$.

Thus by Lemma 7, p. 36, of [5] we have:

CoRollary 3.7. Compatible congruences are permutable.

By Theorem 3.5 every congruence compatible with $S$ is determined by its kernel. Since, by Theorem 3.4, $\Theta_{\rho}$ is compatible with $S$ for any $A-P$ congruence $\rho$ on $S$ we know that $\Theta_{\rho}$ is determined by its kernel. By the following lemma, $\Theta_{\rho}$ is also uniquely determined by ker $\rho$.

Lemma 3.8. Let $\rho$ be an $A-P$ congruence on $S$. Then $x \in \operatorname{ker} \rho$ if and only if $L R(x) \in \operatorname{ker} \Theta_{\rho}$.

Proof. Let $x \in \operatorname{ker} \rho$. Then $x \rho 0 \Rightarrow x y \rho 0$ for any $y \in S$. Thus $R(x / \rho)=S / \rho$ so that $L R(x / \rho)=L(S / \rho)=(0 / \rho)$, i.e., $L R(x) \in \operatorname{ker} \Theta_{\rho}$. If we let $L R(x) \in \operatorname{ker} \Theta_{\rho}$ then $L R(x / \rho)=(0 / \rho)$. Thus $R(x / \rho)=R L R(x / \rho)=$ $R(0 / \rho)=S / \rho$ which gives $x / \rho=0 / \rho$ and we have $x \in \operatorname{ker} \rho$.

That $\Theta$ should be determined completely by $\operatorname{ker} \rho$ is unexpected since an $A-P$ congruence need not be determined by its kernel. For clearly the congruence $\omega$ given by $x \omega y$ if and only if $x=y$ is an $A-P$ congruence with kernel $\{0\}$ as is the congruence $\rho_{0}$ given by $x \rho_{0} y$ if and only if $\dot{\phi}_{x}=\dot{\phi}_{y}$. Clearly $\rho_{0}$ is not generally equal to $\omega$. It turns out that $\rho_{0}$ is the largest $A-P$ congruence with kernel $\{0\}$. Our next project shall be to start with a congruence $\Theta$ compatible with $S$ and determine the existance of an $A-P$ congruence $\lambda$ on $S$, such that $\Theta=\Theta_{\lambda}$. By lemma 3.8 we shall have to construct $\lambda$ so that $\operatorname{ker} \lambda=\{x \in S: L R(x) \in \operatorname{ker} \Theta\}$.

For any congruence $\Theta$ on $\mathscr{L}(S)$ let $S e / \Theta$ denote the equivalence class of $\mathscr{L}(S) / \Theta$ containing $S e$. 
Lemma 3.9. Let $\Theta$ be a congruence compatible with $S$. For each $x \in S$ define $\Phi_{x} ; \mathscr{L}(S) / \Theta \rightarrow \mathscr{L}(S) / \Theta$ by $(\operatorname{Se} / \Theta) \Phi_{x}=\left(\operatorname{Se} \phi_{x}\right) / \Theta$. Then $\Phi_{x}$ is residuated with residual $\Phi_{x}^{+}$given by $(\mathrm{Se} / \Theta) \Phi_{x}^{+}=\left(\operatorname{Se} \phi_{x}^{+}\right) / \Theta$.

Proof. Clearly $\Phi_{x}$ and $\Phi_{x}^{+}$are well defined since $\Theta$ is compatible with $S$. We shall use Lemma 1.3 (iv), i.e., we shall show that the inverse image of a principal ideal is principal. Let $S f / \Theta \in[(0) / \Theta$, $S e / \Theta] \Phi_{x}^{-1}$. Then $(S f / \Theta) \Phi_{x}=\left(S f \phi_{x}\right) / \Theta=\left(S f \phi_{x}\right) / \Theta \cap S e / \Theta=\left(S f \phi_{x} \cap S e\right) / \Theta$. This gives $S f \phi_{x} \Theta\left(S f \dot{\phi}_{x} \cap S e\right)$ so by compatibility with $S$,

$$
S f \cong S f \dot{\phi}_{x} \phi_{x}^{+} \Theta\left(S f \phi_{x} \cap S e\right) \phi_{x}^{+} \cong S e \phi_{x}^{+} .
$$

Thus in $\mathscr{L}(S) / \Theta, S f / \Theta \subseteq\left(S f \phi_{x} \phi_{x}^{+}\right) / \Theta=\left(S f \phi_{x} \cap S e\right) \phi_{x}^{+} / \Theta \subseteq(S e / \Theta) \Phi_{x}^{+}$, i.e., $[(0) / \Theta, S e / \Theta)] \Phi_{x}^{-1} \subseteq\left[(0) / \Theta,(S e / \Theta) \Phi_{x}^{+}\right]$. Now let $S f / \Theta \subseteq(\operatorname{Se} / \Theta) \Phi_{x}^{+}$. Then

$$
S f / \Theta=S f / \Theta \cap\left[(\operatorname{Se} / \Theta) \Phi_{x}^{+}\right]=S f / \Theta \cap\left(\operatorname{Se} \phi_{x}^{+}\right) / \Theta=\left(S f \cap S e \phi_{x}^{+}\right) / \Theta
$$

i.e., $S f \Theta\left(S f \cap S e \phi_{x}^{+}\right)$. By compatibility with $S$

$$
S f \phi_{x} \Theta\left[\left(S f \cap S e \phi_{x}^{+}\right) \phi_{x}\right] \subseteq S e \phi_{x}^{+} \phi_{x} \leqq S e .
$$

Hence $(S f / \Theta) \Phi_{x}=\left(S f \phi_{x}\right) / \Theta=\left(S f \cap S e \phi_{x}^{+}\right) \phi_{x} / \Theta \cong S e / \Theta$. Therefore,

$$
[(0) / \Theta, S e / \Theta] \Phi_{x}^{-1}=\left[(0) / \Theta,(S f / \Theta) \Phi_{x}^{+}\right]
$$

and by Lemma 1.3 (iv), $\Phi_{x}$ is residuated with residual $\Phi_{x}^{+}$.

For any equivalence relation $E$ on $\mathscr{L}(S)$ we can define a left congruence $\lambda_{E}$ on $S$ by taking $x \lambda_{E} y$ if and only if $\left(S e \phi_{x}\right) E\left(S e \phi_{y}\right)$ for all $S e \in \mathscr{L}(S)$. Similarly, $x \rho_{E} y$ if and only if $\left(S e \phi_{x}^{+}\right) E\left(S e \phi_{x}^{+}\right)$for all $S e \in \mathscr{L}(S)$, defines a right congruence on $S$.

LEMMA 3.10. If $\Theta$ is a congruence compatible with $S$ then $\lambda_{\theta}=\rho_{\theta}$. Thus $\lambda_{\theta}$ is a congruence on $S$.

Proof. By definition $x \lambda_{\theta} y$ if and only if $\Phi_{x}=\Phi_{y}$. But $\Phi_{x}=\Phi_{y}$ if and only if $\Phi_{x}^{+}=\Phi_{y}^{+}$which is equivalent to $x \rho_{\theta} y$.

TheOREm 3.11. Let $\Theta$ be a congruence compatible with $S$. Then $\lambda_{\theta}$ is an $A-P$ congruence on $S$.

Proof. We know that $\lambda_{\theta}$ is an $A-P$ congruence if and only if $L\left(y / \lambda_{\theta}\right) \subseteq L(y) / \lambda_{\theta}$ and $R\left(y / \lambda_{\theta}\right) \subseteq R(y) / \lambda_{\theta}$ for all $y \in S$. We shall start with $x / \lambda_{\theta} \in L\left(y / \lambda_{\theta}\right)$ and show that $x / \lambda_{\theta}=x e / \lambda_{\ominus}$ where $S e=L(y)$. This, of course, is equivalent to $\Phi_{x}=\Phi_{x e}$.

Let $x / \lambda_{\theta} \in L\left(y / \lambda_{\theta}\right)$ so that $x y \in \operatorname{ker} \lambda_{\theta}$. Thus $S f \dot{\phi}_{x y} \Theta S f \dot{\phi}_{0}=(0)$ for all $S f \in \mathscr{L}(S)$. In particular, $S \phi_{x y} \Theta(0)$ so for any $S f \in \mathscr{L}(S)$, 


$$
S f \phi_{x} \cong S \phi_{x} \leqq S \phi_{x} \phi_{y} \phi_{y}^{+}=\left(S \phi_{x y} \phi_{y}^{+}\right) \in(0) \phi_{y}^{+}=L(y)=S e .
$$

Thus $S f \phi_{x}=\left(S f \phi_{x} \cap S \phi_{x y} \phi_{x}^{+}\right) \Theta\left(S e \cap S f \phi_{x}\right)$. Now if $S g \subseteq S e$ then $g=g e$ so $S g \phi_{e}=L R(g e)=L R(g)=S g$. Thus applying $\phi_{e}$ to both sides of the above gives $S f \phi_{x e}=S f \phi_{x} \phi_{e} \Theta\left(S e \cap S f \phi_{x}\right) \phi_{e}=S e \cap S f \phi_{x}$. By transitivity $S f \phi_{x e} \otimes S f \dot{\phi}_{x}$ and so $x e \lambda_{\theta} x$. Since $x e \in L(y)$ this gives $x / \lambda_{\theta} \in$ $L(y) / \lambda_{\ominus}$.

The argument to show $R\left(y / \lambda_{\theta}\right)=R(y) / \lambda_{\theta}$ is exactly dual to the above but will be included. We have $x / \lambda_{\theta} \in R\left(y / \lambda_{\theta}\right)$ if and only if $y x \in \operatorname{ker} \lambda_{\theta}$. By Lemma 3.10 and the definition of $\rho_{\theta}$ this is equivalent to $S f \phi_{y x}^{+} \Theta S f \phi_{0}^{+}=L(0)=S$ for all $S f \in \mathscr{L}(S)$. In particular $(0) \phi_{y x}^{+} \Theta S$. By Lemma 1.3 (i) $\phi_{y x}^{+}=\phi_{x}^{+} \phi_{y}^{+}$so for any $S f \in \mathscr{L}(S)$ we have

$$
S f \phi_{x}^{+} \supseteqq(0) \phi_{x}^{+} \supseteqq(0) \phi_{x}^{+} \phi_{y}^{+} \phi_{y}=(0) \phi_{y x}^{+} \phi_{y} \Theta S \phi_{y} \text {. }
$$

Thus $S f \phi_{x}^{+}=\left(S f \phi_{x}^{+} \bigvee(0) \phi_{y x}^{+} \phi_{y}\right) \Theta\left(S \phi_{y} \bigvee S f \phi_{x}^{+}\right)$. Let $e S=R(y)$ and note that $S \phi_{y}=L R(y)=L(e)$. Now $L(e) \cong S g$ implies $e S=R L(e) \supseteqq R(g)=$ $g^{r} S$ so $g^{r}=e g^{r}$. Thus $S g \phi_{e}^{+}=L\left(e g^{r}\right)=L\left(g^{r}\right)=L R(g)=S g$. Since $L(e)=S \phi_{y} \subseteq S \phi_{y} \bigvee S f \phi_{x}^{+}$, applying $\phi_{e}^{+}$to both sides of the above gives ; $S f \dot{\phi}_{e x}^{+}=S f \phi_{x}^{+} \phi_{e}^{+} \theta\left(S \phi_{y} \bigvee S f \phi_{x}^{+}\right) \phi_{e}^{+}=S \phi_{y} \bigvee S f \phi_{x}^{+}$. By transitivity $S f \phi_{x}^{+} \Theta S f \phi_{e x}^{+}$so by Lemma $3.10 x \lambda_{\theta} e x$. Since $e x \in R(y)$ this gives $x / \lambda_{\theta} \in R(y) / \lambda_{\theta}$. Thus $\lambda_{\theta}$ is an $A-P$ congruence.

By Theorem 3.11 every congruence $\Theta$ compatible with $S$ gives rise to an $A-P$ congruence $\lambda_{\theta}$ on $S$.

Lemma 3.12. Let $\Theta$ be a congruence compatible with $S$. Then $x \in \operatorname{ker} \lambda_{\theta}$ if and only if $L R(x) \in \operatorname{ker} \Theta$.

Proof. Let $x \in \operatorname{ker} \lambda_{\theta}$, i.e., $S e \phi_{x} \Theta S e \phi_{0}=(0)$ for all $S e \in \mathscr{L}(S)$. Taking $S e=S$ gives $L R(x) \in \operatorname{ker} \Theta$. Let $L R(x) \in \operatorname{ker} \Theta$. The for any $S e \in \mathscr{L}(S) S e \dot{\phi}_{x} \subseteq S \dot{\phi}_{x}=L R(x) \Theta(0)=S e \phi_{0}$ so $x \in \operatorname{ker} \lambda_{\theta}$.

THEOREM 3.13. Let $\Theta$ be a congruence compatible with $S$ and $\rho=\lambda_{\theta}$. Then $\Theta_{\rho}=\Theta$.

Proof. By Theorem $3.11 \rho$ is an $A-P$ congruence so by Theorem $3.4 \Theta_{\rho}$ is compatible with $S$. By Lemma 3.8 and $3.12 \operatorname{ker} \Theta_{\rho}=\operatorname{ker} \Theta$. Thus by Theorem $3.5 \Theta_{\rho}=\Theta$.

We now show that $\lambda_{\theta}$ is the largest $A-P$ congruence which induces $\Theta$.

CoRollary 3.14. Let $\Theta$ be a congruence compatible with $S$. If $\rho$ is an $A-P$ congruence on $S$ such that $\operatorname{ker} \rho=\operatorname{ker} \lambda_{\theta}$, then $\rho \leqq \lambda_{\theta}$. 


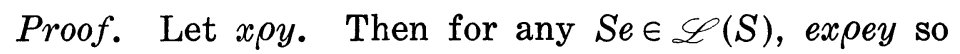

$$
\left(S e \phi_{x}\right) \theta_{\rho}=L R((e x) / \rho)=L R((e y) / \rho)=\left(S e \phi_{y}\right) \theta_{\rho} .
$$

Thus $S e \phi_{x} \Theta_{\rho} S e \phi_{y}$ and since $\Theta_{\rho}=\Theta$ this gives $x \lambda_{\theta} y$.

4. Compatible ideals. In this section ideals which are kernels of congruences compatible with $S$ are characterised. Clearly if $\Theta$ is a congruence compatible with $S$ and $J=\operatorname{ker} \Theta$ then $J \phi_{x} \subseteq J$ for all $x \in S$. Since $\phi_{x}$ preserves join (Lemma 1.3 (iii)) the following is clear.

Lemma 4.1. Let $J$ be an ideal of $\mathscr{L}(S)$ such that $J \phi_{x} \subseteq J$ for all $x \in S$. Define a relation $R$ on $\mathscr{L}(S)$ by Se $R$ Sf if and only if there is an $S g \in J$ such that $S e \bigvee S g=S f \bigvee S g$. Then $R$ is an equivalence relation and $S e R S f \Longrightarrow\left(S e \phi_{x}\right) R\left(S f \phi_{x}\right)$ for all $x \in S$.

In order to find an additional condition on $J$ which will assure that the relation $R$ defined in Lemma 4.1 is compatible with $S$, it will be valuable to look at certain residuated maps on the lattice $I(\mathscr{L}(S))$ of all ideals of $\mathscr{L}(S)$.

Lemma 4.2. For each $x \in S$ let $\hat{\phi}_{x}: I(\mathscr{L}(S) \rightarrow I(\mathscr{L}(S))$ be given by $I \hat{\phi}_{x}=\left\{S e \in \mathscr{L}(S): S e \subseteq S f \phi_{x}\right.$ for some $\left.S f \in I\right\}$. Then $\hat{\phi}_{x}$ is residuated with residual $\hat{\phi}_{x}^{+}$given by $I \hat{\phi}_{x}^{+}=\left\{S e \in \mathscr{L}(S): S e \leqq S f \phi_{x}^{+}\right.$for some $S f \in I\}$.

Proof. Clearly $I \hat{\phi}_{x}$ and $I \hat{\phi}_{x}^{+}$are ideals. Also $\hat{\phi}_{x}$ and $\hat{\phi}_{x}^{+}$are clearly isotone. Now since $S f \subseteq S f \phi_{x} \hat{\phi}_{x}^{+}, \quad S f \in I$ implies $S f \in I \hat{\phi}_{x} \hat{\phi}_{x}^{+}$. Thus $I \subseteq I \hat{\phi}_{x} \hat{\phi}_{x}^{+}$. Similarly $S f \in I \hat{\phi}_{x}^{+} \hat{\phi}$ implies $S f \subseteq S g \phi_{x}^{+} \phi_{x}$ for some $S g \in I$. Thus $S f \subseteq S g \phi_{x}^{+} \phi_{x} \subseteq S g \in I$ so we have $I \hat{\phi}_{x}^{+} \hat{\phi}_{x} \subseteq I$.

We will make use of the residuated maps $\hat{\phi}_{x}$ to characterise ideals which are kernels of congruences compatible with $S$.

LeMmA 4.3. Let $J$ be an ideal of $\mathscr{L}(S)$ such that $J \phi_{x} \leqq J$. Then for any $I \in I(\mathscr{L}(S)), I \hat{\phi}_{x}^{+} \bigvee J \subseteq(I \bigvee J) \hat{\phi}_{x}^{+}$.

Proof. Recall that by Lemma 1.3 (iv), for any residuated map $\phi$ on a lattice $L$ and any $a, b \in L, a \phi \leqq b$ if and only if $a \leqq b \phi^{+}$. Now $\left(I \hat{\phi}_{x}^{+} \bigvee J\right) \hat{\phi}_{x}=I \hat{\phi}_{x}^{+} \hat{\phi}_{x} \bigvee J \hat{\phi}_{x} \subseteq I \bigvee J$ since $I \hat{\phi}_{x}^{+} \hat{\phi}_{x} \leqq I$ and $J \hat{\phi}_{x} \subseteq J$. Thus $I \hat{\phi}_{x}^{+} \bigvee J \subseteq(I \bigvee J) \hat{\phi}_{x}^{+}$.

CoRollary 4.4. Let $J$ be an ideal of $\mathscr{L}(S)$ such that $J \phi_{x} \subseteq J$. Then $J \subseteq J \phi_{x}^{+}$and for any $I \in I(\mathscr{L}(S))$ we have 


$$
I \hat{\phi}_{x}^{+} \bigvee J \subseteq I \hat{\phi}_{x}^{+} \bigvee J \hat{\phi}_{x}^{+} \subseteq(I \bigvee J) \hat{\phi}_{x}^{+}
$$

The next theorem indicates what all of this has to do with congruences compatible with $S$.

LEMmA 4.5. Let $\Theta$ be a congruence compatible with $S$ and $J=$ $\operatorname{ker} \Theta$. Then for any $I \in I(\mathscr{L}(S))$, and any $x \in S$,

$$
I \phi_{x}^{+} \bigvee J=I \phi_{x}^{+} \bigvee J \phi_{x}^{+}=(I \bigvee J) \phi_{x}^{+} .
$$

Proof. By Corollary 4.4 we need only show that $(I \bigvee J) \phi_{x}^{+} \subseteq$ $I \hat{\phi}_{x}^{+} \bigvee J$. Thus let $S e \in I \bigvee J$. Then there is an $S f \in I$ and an $S g \in J$ such that $S e \subseteq S f \bigvee S g$. Since $S g \Theta(0)$ we have $S f \bigvee S g \Theta S f$. Thus, by compatibility with $S$, $S e \phi_{x}^{+} \subseteq(S f \bigvee S g) \phi_{x}^{+} \Theta S f \phi_{x}^{+}$. By Theorem 3.5 there is an $S h \in J$ such that $(S f$ V $S g) \phi_{x}^{+} \bigvee S h=S f \phi_{x}^{+} \bigvee S h$. This gives $S e \phi_{x}^{+} \subseteq(S f \bigvee S g) \phi_{x}^{+} \bigvee S h=S f \phi_{x}^{+} \bigvee S h$ so that $S e \phi_{x}^{+} \in I \hat{\phi}_{x}^{+} \bigvee J$. Thus $(I \bigvee J) \hat{\phi}_{x}^{+} \subseteq I \hat{\phi}_{x}^{+} \bigvee J$.

Without further justification we make the following definition.

Definition 4.6. An ideal $J$ of $\mathscr{L}(S)$ is called compatible with $S$ if for all $x \in S, J \phi_{x} \subseteq J$ and, for all $I \in I(\mathscr{L}(S)), I \hat{\phi}_{x}^{+} \bigvee J=(I \bigvee J) \hat{\phi}_{x}^{+}$.

THEOREM 4.7. An ideal $J$ of $\mathscr{L}(S)$ is compatible with $S$ if and only if it is the kernel of a congruence compatible with $S$.

Proof. By Lemma 4.5 the kernel of a congruence compatible with $S$ is an ideal compatible with $S$. Conversly let $J$ be an ideal compatible with $S$ and define $\Theta$ by $S e \Theta S f$ if and only if there is an $S g \in J$ such that $S e \bigvee S g=S f$ V $S g$. By Lemma $4.1 \Theta$ is an equivalence relation such that $S e \Theta S f$ implies $S e \phi_{x} \Theta S f \phi_{x}$ for all $x \in S$. Let $S e$ V $S g=$ $S f \vee S g, S g \in J$, i.e., let $S e \Theta S f$. Note that

$$
(S f \bigvee S g) \phi_{x}^{+} \in([(0), S f] \bigvee J) \hat{\phi}_{x}^{+}=\left[(0), S f \dot{\phi}_{x}^{+}\right] \bigvee J
$$

and $(S e \bigvee S g) \phi_{x}^{+} \in([(0), S e] \bigvee J) \hat{\phi}_{x}^{+}=\left[(0), S e \phi_{x}^{+}\right] \bigvee J$. Thus there are $S h, S h^{\prime} \in J$ such that

$$
S e \phi_{x}^{+} \subseteq(S e \mathrm{~V} S g) \phi_{x}^{+} \subseteq S e \phi_{x}^{+} \bigvee S h
$$

and

$$
S f \phi_{x}^{+} \subseteq(S f \mathrm{~V} S g) \phi_{x}^{+} \subseteq S f \phi_{x}^{+} \bigvee S h^{\prime} .
$$

Thus $S e \phi_{x}^{+} \bigvee S h=(S e \bigvee S g) \phi_{x}^{+} \bigvee S h$ and $S h^{\prime} \bigvee(S f \bigvee S g) \phi_{x}^{+}=S f \phi_{x}^{+} \bigvee S h^{\prime}$. It follows that $S f \phi_{x}^{+}$V $\left(S h\right.$ V $\left.S h^{\prime}\right)=S e \phi_{x}^{+} \bigvee\left(S h\right.$ V $\left.S h^{\prime}\right)$ and since $S h \bigvee S h^{\prime} \in J$ we have $S e \phi_{x}^{+} \Theta S f \phi_{x}^{+}$. Thus by Lemma $3.3 \Theta$ is a con- 
gruence compatible with $S$.

Note that in the proof of Theorem 4.7 the only use made of the hypothesis $(I \bigvee J) \hat{\phi}_{x}^{+}=I \hat{\phi}_{x}^{+} \bigvee J$ was for $I$ a principal ideal. This observation together with Lemma 4.5 gives.

COROLlaRy 4.8. Let $J$ be an ideal such that $J \dot{\phi}_{x} \subseteq J$ for all $x \in S$. Then $J$ is compatible with $S$ if and only if for any principal ideal $I \in I(\mathscr{L}(S))(I \bigvee J) \hat{\phi}_{x}^{+}=I \hat{\phi}_{x}^{+} \bigvee J$ for all $x \in S$.

By Corollary 4.8 the situation with ideals compatible with $S$ is analogus to that with standard ideals. An ideal $J$ of a lattice $L$ is standard if $(I \bigvee J) \wedge K=(I \wedge K) \bigvee(J \wedge K)$ for all $I, K \in I(L)$. By Theorem 2, p. 30, of [5] an ideal is standard if and only if the above holds for all principal ideals $I, K \in I(L)$. This similarity is not surprising since by Corollary 3.6, Theorem 4.7, and Theorem 2 of [5] any ideal compatible with $S$ is a standard ideal. In fact the definition of ideal compatible with $S$ is closely related to the definition of standard ideal. To see this we need the following:

Lemma 4.9. For any $I \in I(\mathscr{L}(S))$ and any $S e \in \mathscr{L}(S)$,

$$
I \cap[(0), S e]=I \hat{\phi}_{e}^{+} \hat{\phi}_{e} .
$$

Proof. Clearly $I \cap[(0), S e]=\{S f \in \mathscr{L}(S): S f \subseteq S g \cap S e$, for some $S g \in I\}$. But $S g \cap S e=S g \hat{\phi}_{e}^{+} \phi_{e}$ so $I \cap[(0), S e]=I \hat{\phi}_{e}^{+} \hat{\phi}_{e}$.

For any ideal for which $J \phi_{x} \subseteq J$ Corollary 4.4 gives

$$
I \hat{\phi}_{x}^{+} \bigvee J \subseteq I \hat{\phi}_{x}^{+} \bigvee J \hat{\phi}_{x}^{+} \subseteq(I \bigvee J) \dot{\phi}_{x}^{+}
$$

for all $I \in I(L)$. Now $I \hat{\phi}_{x}^{+} \bigvee J \hat{\phi}_{x}^{+}=(I \bigvee J) \hat{\phi}_{x}^{+}$implies

$$
\left(I \hat{\phi}_{x}^{+} \bigvee J \hat{\phi}_{x}^{+}\right) \dot{\phi}_{x}=I \hat{\phi}_{x}^{+} \hat{\phi}_{x} \bigvee J \hat{\phi}_{x}^{+} \hat{\phi}_{x}=(I \bigvee J) \hat{\phi}_{x}^{+} \hat{\phi}_{x} .
$$

Taking $x=e$ with $S e \in \mathscr{C}(S)$ and applying Lemma 4.9 this becomes

$$
(I \cap[(0), S e]) \bigvee(J \cap[(0), S e])=(I \bigvee J) \cap[(0), S e] .
$$

Thus if we had required only $I \hat{\phi}_{e}^{+} \bigvee J \hat{\phi}_{e}^{+}=(I \mathrm{~V} J) \hat{\phi}_{e}^{+}$for all $e$ such that $S e \in \mathscr{L}(S)$ we would have $J$ a standard ideal. However, to define an ideal compatible with $S$ we require the stronger condition that $I \hat{\phi}_{x}^{+} \bigvee J=(I \bigvee J) \hat{\phi}_{x}^{+}$and not only for all idempotents $x$ such that $S x \in \mathscr{C}(S)$ but for all $x \in S$.

5. Compatible elements. An element $a$ of a lattice $L$ is called standard if $x \wedge(a \bigvee y)=(x \wedge a) \bigvee(x \wedge y)$ for all $x, y \in L$. By Lemma 4 , p. 32 of [5] an element is standard if and only if the principal 
ideal it generates is a standard ideal.

Definition 5.1. An element $S e$ of $\mathscr{C}(S)$ is compatible with $S$ if $[(0), S e]$ is an ideal compatible with $S$. Let $\Theta_{S e}$ denote the congruence compatible with $S$ having [(0), $S e]$ as kernel.

Note that by Corollary 3.6 every element compatible with $S$ is a standard element of $\mathscr{C}(S)$.

It will be convenient to look at co-kernels of congruences compatible with $S$.

Lemma 5.2. Let $\Theta$ be a congruence compatible with $S$. Then $L R(x) \in \operatorname{ker} \Theta$ if and only if $L(x) \in \operatorname{co-} \operatorname{ker} \Theta$.

Proof. Let $S f=L R(x) \in \operatorname{ker} \theta$. Then $S f \dot{\phi}_{x}^{+} \theta(0) \dot{\phi}_{x}^{+}=L(x)$. But $S f \phi_{x}^{++}=L\left(x f^{r}\right)$ and since $f^{r} S=R(f)=R(S f)=R L R(x)=R(x)$ we have $S f \dot{\phi}_{x}^{+}=L(0)=S$. Thus $L(x) \in \operatorname{co-ker} \Theta$. Conversly let $L(x) \Theta S$ and note that $L(x) \dot{\phi}_{x}=L R L(x) \dot{\phi}_{x}=L R\left(x^{l}\right) \dot{\phi}_{x}=L R\left(x^{l} x\right)=(0)$. Thus

$$
(0)=L(x) \phi_{x} \Theta S \phi_{x}=L R(x), \quad \text { i.e., } \quad L R(x) \in \operatorname{ker} \Theta .
$$

Lemma 5.3. Let Se be compatible with $S$. Then $S e^{l}$ is a complement of $S e$ and $\left[S e^{l}, S\right]=\operatorname{co-ker} \Theta_{S e}$.

Proof. Clearly $S e \cap S e^{l}=(0)$. By Lemma 5.2, $S e^{l} \Theta_{S e} S$ so, by Theorem 3.5, $S e^{l} \bigvee S e=S$. Thus we clearly have $\left[S e^{l}, S\right] \leqq \operatorname{co-ker} \Theta_{S e}$. Let $S f \in \operatorname{co-ker} \Theta_{S e}$. Then $S f \bigvee S e=S$ and since $S e$ is standard we have $S e^{l}=S e^{l} \cap(S f \bigvee S e)=\left(S e^{l} \cap S f^{\prime}\right) \bigvee\left(S e^{l} \cap S e\right)=S e^{l} \cap S f$. Thus $S e^{l} \cong S f$, i.e., co-ker $\Theta_{S e}=\left[S e^{l}, S\right]$.

We now wish to characterise elements compatible with $S$.

Lemma 5.4. Let Se be compatible with $S$. Then e is central in $S$ and $e S=R L(e)$.

Proof. By Lemma 5.2, $S e^{l} \in \operatorname{co-ker} \Theta_{S e}$. Since $S e^{l}=L R\left(e^{l}\right)=L\left(e^{l r}\right)$ applying Lemma 5.2 again gives $L R\left(e^{l r}\right) \in \operatorname{ker} \Theta_{S e}$, i.e., $L R\left(e^{l r}\right) \leqq S e$. Thus $e^{l r}=e^{l r} e$. But $e^{l} e=0$ implies $e \in R\left(e^{l}\right)$ so $e=e^{l r} e$. Thus $e=$ $e^{l r}$ so $e S=R\left(e^{l}\right)=R L(e)$. By Lemma 5.3, $S e^{l} \phi_{x}^{+} \supseteqq S e^{l}$ and $S e^{l} \dot{\phi}_{x}^{+}=$ $L\left(x e^{l r}\right)=L(x e)$. Thus $R L(x e) \subseteq R\left(e^{l}\right)=R L(e)=e S$ so $x e=e x e$. But $S e \dot{\phi}_{x} \subseteq S e$ so $e x=e x e=x e$, i.e., $e$ is central in $S$.

We can use any central idempotent of $S$ to induce an $A-P$ congruence on $S$ as follows:

Lemma 5.5. Let $e$ be central in $S$ and define a relation $\rho$ on $S$ 
by xpy if and only if $x e=y e$. Then $\rho$ is an $A-P$ congruence on $S$ and $\operatorname{ker} \rho=S e^{l}$.

Proof. Clearly $\rho$ is a congruence on $S$. Let $y / \rho \in L(x / \rho)$. Then $0 / \rho=(y / \rho)(x / \rho)=(y x) / \rho$ so $y x e=0$. But $y x e=(y e) x$ so $y e \in L(x)$. Thus $y e=(y e) e$ gives $y / \rho=(y e) / \rho \in L(x) / \rho$. Similarly $R(x / \rho) \subseteq R(x) / \rho$. Clearly $x / \rho=0 / \rho$ if and only if $x \in L(e)=S e^{l}$.

Lemma 5.6. If e is central in $S$ then $S e^{l}$ is compatible with $S$.

Proof. Since $e$ is central $x \rho y$ if and only if $x e=y e$ is an $A-P$ congruence with kernel $S e^{l}$. By Lemma 3.8, $L R(x) \in \operatorname{ker} \Theta_{\rho}$ if and only if $x \in S e^{l}$. But $x \in S e^{l}$ if and only if $x=x e^{l}$ if and only if $L R(x) \subseteq S e^{l}$. Thus $\operatorname{ker} \Theta_{\rho}=\left[(0), S e^{l}\right]$ so that $S e^{l}$ is compatible with $S$.

We can now characterise elements compatible with $S$ as follows :

Theorem 5.7. Let $S e \in \mathscr{L}(S)$. Then $S e$ is compatible with $S$ if and only if $e$ is central in $S$.

Proof. Let $e$ be central in $S$. By Lemma 5.6, $S e^{l}$ is compatible with $S$. Now $L(e)=R(e)$ so $S e^{l}=e^{r} S$. Thus $e^{l}=e^{r} e^{l}=e^{r}$. By Lemma 5.6, $S e^{l}=S e^{r}$ compatible with $S$ gives $S e^{r l}$ compatible with $S$. But $S e^{r l}=L R\left(e^{r l}\right)=L R(e)=S e$. Thus $S e$ is compatible with $S$. The converse is Lemma 5.4.

Note that $S e$ is compatible with $S$ if and only if $S e^{l}$ is compatible with $S$. Thus, by Lemma 5.3, if either $S e$ or $S e^{l}$ is compatible with $S$ then $S e$ and $S e^{l}$ are standard elements of $\mathscr{L}(S)$ which are complements. Thus by Theorem 7.3, p. 300, of [6] we have.

THEOREM 5.8. If either Se or $S e^{l}$ is compatible with $S$ then:

(i) Both Se and $S e^{l}$ are compatible with $S$.

(ii) Both $S e$ and $S e^{l}$ are central in $\mathscr{f}(S)$.

(iii) $\Theta_{S e}$ and $\Theta_{S e^{l}}$ are complements in $\Theta\left(S^{=}(S)\right)$.

Corollary 5.9. Let $S e \in \mathscr{f}(S)$. Then if e is central in S, Se is central in $\mathcal{E}(S)$.

5. The lattice of compatible congruences. From the formula for meet and join in $\Theta(L)$ (see Theorem 1.7) it is clear that both the meet and the join of any family of congruences compatible with $S$ are congruences compatible with $S$. Thus, applying Theorem 1.8, we have. 
THEOREM 6.1. The lattice $\Theta_{S}(\mathscr{L}(S))$ of all congruence compatible with $S$ is a subcomplete sublattice of $\Theta(\mathscr{L}(S))$. Thus $\Theta_{S}(\mathscr{L}(S))$ is an uppercontinuous distributive lattice.

It follows from [1], Theorem 15, p. 147, that $\Theta_{S}(\mathscr{L}(S))$ is pseudocomplemented. If $\Theta \in \Theta_{S}(\mathscr{L}(S))$ we shall use $\Theta^{*}$ to denote the pseudocomplent of $\Theta$ in $\Theta(\mathscr{L}(S))$ and $\Theta^{\prime}$ to denote the pseudo-complement of $\Theta$ in $\Theta_{S}(\mathscr{L}(S))$.

In [9], Theorem 4.17 (iii), it is shown that for a complete relatively complemented lattice $L, \theta(L)$ is a Stone lattice in the sense that every pseudo-complement has a complement. The remainder of this section is devoted to showing that for suitable choice of $S$, $\Theta_{S}(\mathscr{L}(S))$ is a Stone lattice.

We first look at the left and right annihilators of the kernel of an $A-P$ congruence.

Lemma 6.2. Let $\rho$ be an $A-P$ congruence on $S$ and $J=\operatorname{ker} \rho$. Then $L(J)=R(J)$.

Proof. Let $x \in J$ and $y \in L(J)$. If $z \in J$ then $x y z=0$. Thus $J \cong R(x y)$ so that $L(J) \supseteqq L R(x y)$. Let $L R(x y)=S f$ and note that $f \in L(J)$. Since $J$ is an ideal, $x y \in J$, i.e., $x y / \rho=0 / \rho$. Thus

$$
f / \rho \in L R(x y) / \rho=L R(x y / \rho)=L R(0 / \rho)=(0 / \rho)
$$

so $f \in J$. But then we have $f \in J \cap L(J)$ so $f=f^{2}=0$. This gives $L R(x y)=(0)$ which implies $x y=0$. Thus $L(J) \cong R(J)$. By symmetry $R(J) \subseteq L(J)$ so $R(J)=L(J)$.

Recall that a semigroup $S$ is a complete Baer semigroup if the left and right annihilators of an arbitrary subset of $S$ are principal left and right ideals generated by idempotents. Also (Theorem 1.6) as $S$ ranges over all complete Baer semigroups $\mathscr{L}(S)$ ranges over all complete lattices.

Lemma 6.3. Let $S$ be a complete Baer semigroup, $\Theta$ a congruence compatible with $S$, and $S e=\cap \operatorname{co-ker} \Theta$. Then $S e$ is compatible with $S$.

Proof. Let $J=\operatorname{ker} \lambda_{\theta}$. By Lemmas 5.2 and 3.12, $x \in J$ if and only if $L(x) \in \operatorname{co-ker} \Theta$. Thus $L(J) \subseteq S e$ since $L(J) \subseteq L(x)$ for all $x \in J$. But $S e \subseteq L(x)$ for all $x \in J$ gives $S e \subseteq L(J)$. Thus $S e=L(J)$. Now by Lemma $6.2, L(J)=R(J)$ and since $S$ is a complete Baer semigroup there is an idempotent $f \in S$ such that $f S=R(J)$. Then 
$f S=S e$ so $e=f e=f$. Since $S e=e S$ is an ideal we have $e x=e x e=$ $x e$ for all $x \in S$. Thus $e$ is central in $S$ so by Theorem 5.7, $S e$ is compatible with $S$.

We can now characterise the kernel of the pseudo-complement of a congruence compatible with a complete Baer semigroup.

THEOREM 6.4. Let $S$ be a complete Baer semigroup and $\Theta$ a congruence compatible with $S$. Then $\operatorname{ker} \Theta^{*}$ is a principal ideal generated by an element of $\mathscr{L}(S)$ which is compatible with $S$.

Proof. Let $S e=\cap \operatorname{co-ker} \Theta$ and $J=\operatorname{ker} \lambda_{\theta}$. By Lemma 6.3, Se is compatible with $S$. But $S e=L(J)=R(J)$ and $x \in J$ if and only if $L R(x) \in \operatorname{ker} \Theta$ gives $S e \cap S f=(0)$ for all $S f \in \operatorname{ker} \Theta$. Thus ker $\Theta_{S e} \cap \operatorname{ker} \Theta=(0)$ so by Theorem 3.5, $\Theta_{S e} \Lambda \Theta=\omega$. By definition of pseudo-complement we have $\Theta_{S e} \leqq \Theta^{*}$ so $[(0), S e]=\operatorname{ker} \Theta_{S e} \subseteq \operatorname{ker} \Theta^{*}$. Now let $S g \in \operatorname{co-ker} \Theta$ and $S f \in \operatorname{ker} \Theta^{*}$. Then $(S f \cap S g) \Theta(S f \cap S)=S f$ and $(S f \cap S g) \Theta^{*}(0)$. Since $(0) \Theta^{*} S f$ we have $(S f \cap S g) \equiv S f\left(\Theta \wedge \Theta^{*}\right)$. This gives $S f \cap S g=S f$ so $S f \subseteq S g$. Thus $S f \subseteq S e$ and $\operatorname{ker} \Theta^{*} \subseteq$ $[(0), S e]$. We, therefore, have $\operatorname{ker} \Theta^{*}=[(0), S e]$ and since $S e$ is compatible with $S$ this completes the proof.

We clearly have $\Theta^{\prime} \subseteq \Theta^{*}$. Since $\operatorname{ker} \Theta^{*}$ is a principal ideal generated by an element $S e$ compatible with $S$, it is clear that $\Theta^{\prime}=\Theta_{S e}$. By Theorem 5.8, $S e^{l}$ is compatible with $S$ and $\Theta_{S e^{l}}$ is a complement of $\Theta_{S e}$ in $\theta_{S}(\mathscr{C}(S))$.

TheOREM 6.5. Let $S$ be a complete Baer semigroup. Then $\Theta_{S}(\mathscr{L}(S))$ is a Stone lattice.

\section{BIBLIOGRAPHY}

1. G. Birkhoff, Lattice theory, Amer. Math. Soc. Colloq. Publ. XXV, 2nd ed., Providence, R. I., 1948.

2. R. Croisot, Applications résiduées, Paris, Ecole normale Superieure, Annales Scientifiques 73 (1956), 453-474.

3. C. J. Everett, Closure operators and Galois theory in lattices, Trans. Amer. Math. Soc. 55 (1944), 514-525.

4. N. Funayama and T. Nakayama, On the distributivity of a lattice of lattice congruences, Proc. Imp. Acad. Tokyo 18 (1942), 553-554.

5. G. Grätzer and E. Schmidt, Standard ideals in lattices, Acta. Math. Acad. Sci. Hung. 12 (1961), 17-86.

6. M. F. Janowitz, A characterisation of standard ideals, Acta. Math. Acad. Sci. Hung. 16 (1965), 289-301.

7. — A semigroup approach to lattices, Canad. J. Math. 18 (1966), 1212-1223.

8. — Baer semigroups, Duke Math. J. 23 (1963), 85-95. 
9. - Section semicomplemented lattices (to appear)

10. S. Maeda, On a ring whose principal right ideals generated by idempotents form a lattice, Hiroshima J. of Sci. 24 (1960), 509-525.

11. O. Ore, Galois connexions, Trans. Amer. Math Soc. 55 (1944), 493-513.

Received June 21, 1968. The results presented here were included in the author's doctoral dissertation presented at the University of New Mexico and were obtained while a member of the faculty of Smith college. The author wishes to express his gratitude to M. F. Janowitz for his generous help and guidance.

SANDIA CORPORATION 


\section{PACIFIC JOURNAL OF MATHEMATICS}

\section{EDITORS}

\author{
H. ROYDEN \\ Stanford University \\ Stanford, California \\ R. R Phelps \\ University of Washington \\ Seattle, Washington 98105
}

\author{
J. Dugundui \\ Department of Mathematics \\ University of Southern California \\ Los Angeles, California 90007
}

RICHARD ARENS

University of California

Los Angeles, California 90024

\section{ASSOCIATE EDITORS}
E. F. BeCKENBACH
B. H. NeUmanN
F. WolF
K. YosidA

\section{SUPPORTING INSTITUTIONS}

\author{
UNIVERSITY OF BRITISH COLUMBIA \\ CALIFORNIA INSTITUTE OF TECHNOLOGY \\ UNIVERSITY OF CALIFORNIA \\ MONTANA STATE UNIVERSITY \\ UNIVERSITY OF NEVADA \\ NEW MEXICO STATE UNIVERSITY \\ OREGON STATE UNIVERSITY \\ UNIVERSITY OF OREGON \\ OSAKA UNIVERSITY \\ UNIVERSITY OF SOUTHERN CALIFORNIA
}

\author{
STANFORD UNIVERSITY \\ UNIVERSITY OF TOKYO \\ UNIVERSITY OF UTAH \\ WASHINGTON STATE UNIVERSITY \\ UNIVERSITY OF WASHINGTON

$* * * * *$
AMERICAN MATHEMATICAL SOCIETY
CHEVRON RESEARCH CORPORATION
TRW SYSTEMS
NAVAL WEAPONS CENTER

The Supporting Institutions listed above contribute to the cost of publication of this Journal, but they are not owners or publishers and have no responsibility for its content or policies.

Mathematical papers intended for publication in the Pacific Journal of Mathematics should be in typed form or offset-reproduced, double spaced with large margins. Underline Greek letters in red, German in green, and script in blue. The first paragraph or two must be capable of being used separately as a synopsis of the entire paper. It should not contain references to the bibliography. Manuscripts, in duplicate if possible, may be sent to any one of the four editors. Please classify according to the scheme of Math. Rev. 36, 1539-1546. All other communications to the editors should be addressed to the managing editor, Richard Arens, University of California, Los Angeles, California, 90024.

50 reprints are provided free for each article; additional copies may be obtained at cost in multiples of 50 .

The Pacific Journal of Mathematics is published monthly. Effective with Volume 16 the price per volume (3 numbers) is $\$ 8.00$; single issues, $\$ 3.00$. Special price for current issues to individual faculty members of supporting institutions and to individual members of the American Mathematical Society: $\$ 4.00$ per volume; single issues $\$ 1.50$. Back numbers are available.

Subscriptions, orders for back numbers, and changes of address should be sent to Pacific Journal of Mathematics, 103 Highland Boulevard, Berkeley, California, 94708.

PUBLISHED BY PACIFIC JOURNAL OF MATHEMATICS, A NON-PROFIT CORPORATION

Printed at Kokusai Bunken Insatsusha (International Academic Printing Co., Ltd.), 7-17. Fujimi 2-chome, Chiyoda-ku, Tokyo, Japan. 


\section{Pacific Journal of Mathematics}

Vol. 28, No. 3

May, 1969

Jon F. Carlson, Automorphisms of groups of similitudes over $F_{3} \ldots \ldots \ldots$

W. Wistar (William) Comfort, Neil Hindman and Stelios A. Negrepontis,

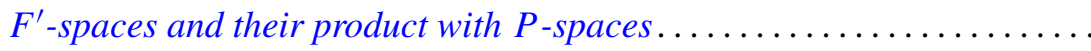

Archie Gail Gibson, Triples of operator-valued functions related to the unit

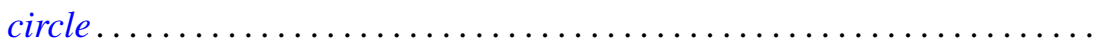

David Saul Gillman, Free curves in $E^{3}$

E. A. Heard and James Howard Wells, An interpolation problem for

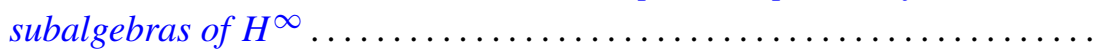

Albert Emerson Hurd, A uniqueness theorem for weak solutions of symmetric

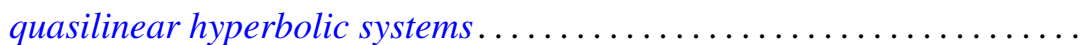

E. W. Johnson and J. P. Lediaev, Representable distributive Noether

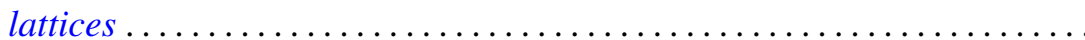

David G. Kendall, Incidence matrices, interval graphs and seriation in

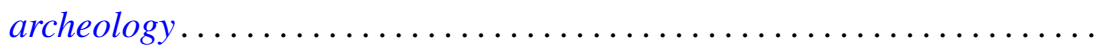
565

Robert Leroy Kruse, On the join of subnormal elements in a lattice ....... 571

D. B. Lahiri, Some restricted partition functions; Congruences modulo 3 .... 575

Norman D. Lane and Kamla Devi Singh, Strong cyclic, parabolic and conical differentiability........................................

William Franklin Lucas, Games with unique solutions that are

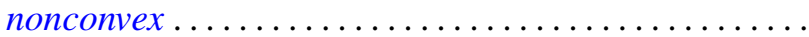

Eugene A. Maier, Representation of real numbers by generalized geometric series.

Daniel Paul Maki, A note on recursively defined orthogonal polynomials ...

Mark Mandelker, $F^{\prime}$-spaces and z-embedded subspaces ...

James R. McLaughlin and Justin Jesse Price, Comparison of Haar series with gaps with trigonometric series

Ernest A. Michael and A. H. Stone, Quotients of the space of irrationals ....

William H. Mills and Neal Zierler, On a conjecture of Golomb ...

J. N. Pandey, An extension of Haimo's form of Hankel convolutions ...

Terence John Reed, On the boundary correspondence of quasiconformal mappings of domains bounded by quasicircles...

Haskell Paul Rosenthal, A characterization of the linear sets satisfying Herz's criterion.

George Thomas Sallee, The maximal set of constant width in a lattice...

I. H. Sheth, On normaloid operators

James D. Stasheff, Torsion in BBSO ...

Billy Joe Thorne, A - P congruences on Baer semigroups.

Robert Breckenridge Warfield, Jr., Purity and algebraic compactness for

modules... 